\title{
Front Matter: Volume 8257
}

, "Front Matter: Volume 8257," Proc. SPIE 8257, Optical Components and Materials IX, 825701 (2 April 2012); doi: 10.1117/12.931212

SPIE. Event: SPIE OPTO, 2012, San Francisco, California, United States 


\title{
PRO CEEDINGS OF SPIE
}

\section{Optical Components and Materials IX}

\author{
Shibin J iang \\ Michel J. F. Digonnet \\ J. Christopher Dries \\ Editors
}

\section{5-26 J anuary 2012 \\ San Francisco, Califomia, United States}

Sponsored and Published by

SPIE 
The papers included in this volume were part of the technical conference cited on the cover and title page. Papers were selected and subject to review by the editors and conference program committee. Some conference presentations may not be available for publication. The papers published in these proceedings reflect the work and thoughts of the authors and are published herein as submitted. The publisher is not responsible for the validity of the information or for any outcomes resulting from reliance thereon.

Please use the following format to cite material from this book:

Author(s), "Title of Paper," in Optical Components and Materia Is IX, edited by Shib in J iang, Michel J. F. Digonnet, J . Christopher Dries, Proceedings of SPIE Vol. 8257 (SPIE, Bellingham, WA, 2012) Artic le CID Number.

ISSN 0277-786X

ISBN 9780819489005

Published by

SPIE

P.O. Box 10, Bellingham, Wa shington 98227-0010 USA

Telephone +1 3606763290 (Pacific Time)· Fax +1 3606471445

SPIE.org

Copyright $\odot 2012$, Society of Photo-Optic al Instrumentation Engineers

Copying of material in this book for intemal or personal use, or for the intemal or personal use of specific clients, beyond the fair use provisions granted by the U.S. Copyright Law is authorized by SPIE subject to payment of copying fees. The Transactional Reporting Service base fee for this volume is $\$ 18.00$ per article (or portion thereof), which should be paid directly to the Copyright Clearance Center (CCC), 222 Rosewood Drive, Danvers, MA 01923. Payment may also be made electronically through CCC Online at copyright.com. Other copying for republication, resale, advertising or promotion, or any form of systematic or multiple reproduction of any material in this book is prohibited except with permission in writing from the publisher. The CCC fee code is $0277-786 \mathrm{X} / 12 / \$ 18.00$.

Printed in the United States of America.

Public ation of record for individual papers is online in the SPIE Digital Library.

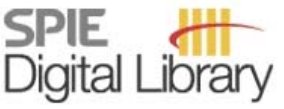

SPIEDigitallibrary.org

Paper Numbering: Proceedings of SPIE follow an e-First public ation model, with papers published first online and then in print and on CD-ROM. Papers are published as they a re submitted and meet public ation criteria. A unique, consistent, permanent citation identifier (CID) number is assigned to each artic le at the time of the first public ation. Utilization of CIDs a llows artic les to be fully citable as soon as they are published online, and connects the same identifier to all online, print, and electronic versions of the public ation. SPIE uses a six-digit CID artic le numbering system in which:

- The first four digits correspond to the SPIE volume number.

- The last two digits indicate publication order within the volume using a Base 36 numbering system employing both numerals and letters. These two-number sets start with $00,01,02,03,04$, $05,06,07,08,09,0 A, 0 B \ldots$ 0Z, followed by 10-1Z, 20-2Z, etc.

The CID numberappears on each page of the manuscript. The complete citation is used on the first page, and an abbreviated version on subsequent pages. Numbers in the index correspond to the last two digits of the six-digit CID number. 


\section{Contents}

ix Conference Committee

xi Spinoptics: Spin degeneracy removal in nanostructures (Plenary Paper) [8269-100]

V. Kleiner, N. Shitrit, E. Hasman, Tec hnion-Israel Institute of Technology (Israel)

\section{SESSION 1 MATERIALSPECTROSCOPY I}

825702 Sensitization of erbium through silic on nanocrystals in silic on rich oxide [8257-02]

M. Q. Huda, Bangladesh Univ. of Engineering and Technology (Bangladesh)

825703 Recent advancements in multiband IR sensor windows [8257-03]

S. Bayya, J. Sanghera, W. Kim, G. Villa lobos, U.S. Naval Research Lab. (United States);

I. Aggarwal, Sotera Defense Solutions, Inc. (United States)

825704 Development of Praseodymium doped fluoride waveguide [8257-04]

M. Olivier, Sc iences Chimiques de Rennes, CNRS, Univ. de Rennes 1 (France); J .-L Doua lan, P. Camy, CIMAP, ENSICAEN (France); H. Lhermite, IEIR-Mic roelectronique, Univ. de Rennes

1 (France); J.-L Adam, V. Nazabal, Sciences Chimiquesde Rennes, CNRS, Univ. de Rennes 1 (France)

825705 A new vision of photodarkening in $\mathbf{Y b}^{3+-d o p e d ~ f i b e r s ~[8257-05] ~}$

R. Peretti, Univ. Lyon 1, CNRS and Ecole Centrale de Lyon (France); C. Gonnet, Draka (France); A.-M. J urd yc, Univ. Lyon 1, CNRS (France)

825706 Spectroscopic study of $\mathrm{Nd}^{3+}$ ions in $0.8 \mathrm{CaSiO}{ }_{3}-\mathrm{O}_{-2} \mathrm{Ca}_{3}\left(\mathrm{PO}_{4}\right)_{2}$ eutectic glass-ceramics [8257-37]

D. Sola, Ctr. de Físic a de Materia les (Spain); R. Balda, Ctr. de Física de Materiales (Spa in) and Univ. del País Vasco (Spa in); J I I. Peña, Univ. de Zaragoza (Spain); J . Femández, Ctr. de Física de Materia les (Spain) and Univ. del País Vasco (Spain)

825707 Novel calculation for cross-relaxation energy transfer parameter applied on thulium highly doped tellunite glasses [8257-07]

M. Taher, H. Gebavi, S. Taccheo, Swansea Univ. (United Kingdom); D. Milanese, Politecnico di Torino (Italy); R. Balda, Univ. del País Vasco and Ctr. de Físic a de Materiales (Spain)

825708 One- and two-photon pumped random laseraction in Rhodamine B doped di-ureasil hybrids [8257-08]

S. García-Revilla, Univ. del País Vasco (Spa in); E. Pecoraro, Univ. de Aveiro (Portugal); R. Balda, Univ. del País Vasco (Spa in) and Ctr. de Física de Materiales (Spa in); L. Carlos, Univ. de Aveiro (Portugal); J. Femández, Univ. del País Vasco (Spain) and Ctr. de Física de Materiales (Spain) 
825709 Advances in modeling of photonic structures for glass lasers (Invited Paper) [8257-09] F. Prudenzano, L Mescia, P. Bia, A. Di Tommaso, M. De Sario, Politec nico di Bari (Italy)

8257 OA - 2.1 um $\mathbf{T m}^{3+} \mathbf{H o}^{3+}$ co-doped tungsten tellunite single mode fiber laser [8257-10] K. Li, Shanghai Institute of Optics and Fine Mechanics (China); G. Zhang, Sha nghai Institute of Optics and Fine Mechanics (China) and Graduate School of Chinese Academy of Sciences (China); L. Hu, M. Wang, D. Chen, Shanghai Institute of Optic sand Fine Mechanics (China)

8257 OB Er ${ }^{3+}$-doped tellunite mic ro-stuctured fiber: laser generation and optical gain [8257-11] E. F. Chillcce, Univ. Esta dual de Campinas (Bra zil); R. Narro-García, Instituto Politécnico Nacional (CICATA-IPN) (Mexico); J. W. Menezes, Univ. Estadual de Campinas (Bra zil); E. Rodriguez, Instituto Politécnico Nacional (CICATA-IPN) (Mexico); D. Marconi, H. L Fragnito, L C. Barbosa, Univ. Estadual de Campinas (Brazil)

SESSION 4 MICROSTRUCTURED RBERS

8257 0C Recent advances in very highly nonlinear chalcogenide photonic crystal fibers and their applications (Invited Paper) [8257-13]

D. Méchin, L. Brilland, Plate-forme d'Étude et de Recherche sur les Fib res Optiques Spéciales (France); J. Troles, Univ. Européenne de Bretagne (France) and CNRS, Sciences Chimiques de Rennes, Univ. de Rennes 1 (France); T. Chartier, P. Besnard, Univ. Européenne de Bretagne, CNRS Foton (Fra nce); G. Canat, ONERA (France); G. Renversez, CNRS, Institut Fresnel, Univ. d'Aix-Marseille (France)

8257 OD Chalcogenide-tellurite composite mic rostructured optical fibre [8257-15]

T. Kohoutek, Z Duan, H. Kawa shima, X. Yan, T. Suzuki, Toyota Tec hnologic al Institute (J apan); M. Matsumoto, T. Misumi, The Funukawa Denshi Co., Ltd. (J apan); Y. Ohishi, Toyota Technological Institute (J apan)

\section{SESSION 5 FBER LASERS}

8257 OE Broad-band laser emission and optical mode-locking of a Fabry-Perot laser formed between two linearty Chiped Fiber Bragg Gratings [8257-20]

G. A. lordachesc u, X. Q. Wu, J. J acquet, Supélec (France)

8257 OF Tunable and switc hable multi-wavelength fiber laser based on semic onductor optical amplifier and twin-core photonic crystal fiber [8257-21]

B. Kim, J. Han, Y. Chung, Gwangju Institute of Sc ience and Technology (Korea, Republic of) 
8257 0G Recent developments in the fabrication of infrared fiber Bragg gratings [8257-22]

L Busse, U.S. Naval Research Lab. (United States); C. Florea, Sotera Defense Solutions (United States); B. Sha w, J. Sanghera, V. Nguyen, U.S. Naval Research Lab. (United States); G. C hin, Univ. Research Foundation (United States); I. Aggarwal, Sotera Defense Solutions (United States)

$8257 \mathrm{OH}$ Temperature stability in silica based fiber Bragg gratings [8257-23]

C. W. Smelser, S. J. Miha ilov, D. Grobnic, Communic ations Research Ctr. Canada (Canada)

8257 ol Single resonance monolithic Fabry-Perot filters formed by volume Bragg gratings and multilayer dielectric mimors [8257-24]

J. Lumeau, CREOL, The College of Optics and Photonics, Univ. of Central Florida (United States); C. Koc, Institut Fresnel, CNRS (France); O. Mokhun, V. Smimov, OptiG ra te Com. (United States); M. Lequime, Institut Fresnel, CNRS (France); L B. Glebov, CREOL, The College of Optics and Photonics, Univ. of Central Florida (United States)

8257 0] High order mode long-period fiber grating refractive index sensor based on intensity measurement [8257-25]

X. Lan, Missouri Univ. of Science and Technology (United States); Q. Han, Missouri Univ. of Science and Technology (United States) and Tia njin Univ. (China); J . Huang, X. Fang, T. Wei, Z Gao, H. Xiao, Missouri Univ. of Science and Technology (United States)

\section{SESSION 7 OPIICALCOMPONENIS I}

8257 OL El Ectro-active multi-layer fluidic lens of PDMS membrane for optical zooming system [8257-26]

H. Choi, S. W. Schin, Y. H. Won, KAIST (Korea, Republic of)

8257 OM Fexible, fibre-addressable surface-plasmon-resonance chip [8257-27]

F. Chowdhury, K. J. Chau, The Univ. of British Columbia (Canada)

8257 ON Optical attenuation across a metal grid [8257-28]

M. M. Tilleman, Elb it Systems of America (United Sta tes)

825700 Numerical simulation of laser pulse propagation in rare-earth-doped materials [8257-30] K. Beeson, E. Parilov, M. J. Potasek, Simphotek Inc. (United States)

\section{SESSION 8 OPIICALCOMPONENIS II}

8257 OP Compactsilica-on-silic on planar lightwave circ uits for high speed optical signal processing [8257-31]

C. L Callender, P. Duma is, C. Bla nchetiere, S. Jacob, C. Ledderhof, C. W. Smelser, Communic ations Research Ctr. Canada (Canada); K. Yadav, J. Albert, Carleton Univ. (Canada)

$82570 \mathrm{Q}$ Influence of coating stress of diamond-like carbon coating on surface flatness of large gemanium windows [8257-32]

S. R. Yadagin, R. Siew, Qioptiq Singapore Pte Ltd. (Singapore) 
8257 OR Three-phase photoconductive elements for directional free-space optical sensing [8257-33] X. J in, D. Guerrero, J . F. Holzman, The Univ. of British Columbia (Canada)

8257 OS Demonstration of all-optical two bit digital comparator using self-locked Fabry-Perot laser diode [8257-34]

B. Nakami, M. Ra kib-Uddin, Y. H. Won, KAIST (Korea, Republic of)

8257 0T The foc using property of the spiral Fibonacci zone plate [8257-35]

H. T. Da i, Tianjin Univ. (China); Y. J . Lu, A*STAR Institute of Materia Is Research and Engineering (Singapore); X. W. Sun, Tianjin Univ. (China) and Nanyang Technological Univ. (Singapore)

\section{POSTER SESSION}

8257 OU Quantum-comelated photon pair generation in tellunite mic rostructured optical fibers [8257-16]

X. Yan, M. Liao, T. Suzuki, Y. Ohishi, Toyota Technological Institute (J apan)

8257 OV Fabrication of photonic crystals in rare-earth doped chalcogenide glass films forenhanced upc onversion [8257-17]

M. E. Pollard, Univ. of Southampton (United Kingdom); K. J . Knight, The Univ. of Adelaide (Australia); G. J . Parker, D. W. Hewak, M. D. B. Chartton, Univ. of So uthampton (United Kingdom)

8257 OW Passively mode-locked and Q-switched operation in a fiber laser cavity with nomal dispersion [8257-19]

W. Gao, M. Liao, X. Yan, T. Suzuki, Y. Ohishi, Toyota Technological Institute (J apan)

8257 OX Optical power control filters: from laser dazding to damage protection [8257-36]

A. Donval, K. Golding, D. Nevo, T. Fisher, O. Lipman, M. Oron, KiloLambda Technologies, Ltd. (Israel)

8257 OY A dispersion flattened tellunite composite holey fiber[8257-38]

M. Lia o, Z Duan, W. Gao, X. Yan, T. Suzuki, Y. Ohishi, Toyota Technological Institute (J apan)

$82570 Z$ Optimization in Raman+EDFA hybrid amplifiers for WDM systems [8257-39]

M. M. J ardim Martini, Ctr. Federal de Educação Tecnológica de Minas Gera is (Brazil);

C. E. S. Castellani, M. J . Pontes, M. R. N. Ribeiro, Univ. Federal do Espínito Sa nto (Brazil);

H. J . Ka linowski, Univ. Tecnológica Federal do Paraná (Brazil)

$825710 \quad$ Numerical simulation of Nd-fluoride and tellurite solar-pumped fiber lasers [8257-40] T. Suzuki, H. Nasu, Toyota Technologic al Institute (J a pan); S. Mizuno, H. Ito, K. Hasegawa, Toyota Central R\&D Labs., Inc . (J apan); Y. Ohishi, Toyota Technological Institute (J apan)

825711 Novel tellunite-phosphate composite mic rostructured optical fibers for nonlinear applications [8257-41]

T. H. Tong, X. Yan, Z Duan, M. Liao, T. Suzuki, Y. Ohishi, Toyota Tec hnologica I Institute (J a pa n)

825712 Structure light with laser speckle for object contour reconstruction [8257-42]

T.-X. Hua, C.-H. Chen, National Tsing Hua Univ. (Ta iwan); A. Tsa i, W.-K. Liu, Institute for Information Industry (Taiwan) 
825713 Nondestructive testing of electron beam sterilization by means of an optically active marker material [8257-43]

T. Härtling, M. Reitzig, Fraunhofer Institute for Nondestruc tive Testing (Germany); A. Mayer, Gigatag G mbH (Gemany); C. Wetzel, Fraunhofer Institute for Electron Beam and Pla sma Technology (Germany); O. Röder, J. Schreiber, J . O pitz, Fraunhofer Institute for Nondestruc tive Testing (Gemany)

825716 Optical humidity sensor using Polypymole (PPy) [8257-46]

M. A. Armas Alvara do, D. O. Canvalho, G. Rehder, J. Gruber, R. W. C. Li, M. I. Alayo, Univ. de São Paulo (Brazil)

825717 Refractive-index insensitive long-period fiber gratings point-by-point inscribed by $\mathrm{CO}_{2}$ laser for fiber sensors and lasers [8257-47]

Q. Han, Missouri Univ. of Sc ience and Technology (United States) and Tianjin Univ. (China); X. Lan, J. Huang, T. Wei, Z Gao, H. Xiao, Missouri Univ. of Science and Technology (United States)

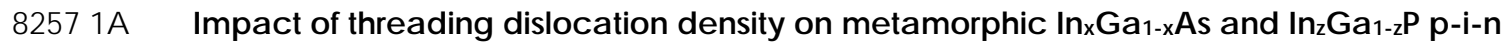
photodetectors on GaAs [8257-51]

K. Swaminathan, T. J . Grassman, L-M. Yang, D. Chmielewski, M. Mills, S. A. Ringel, The Ohio State Univ. (United States)

8257 1B Bend loss insensitive all-solid optical fiber [8257-52]

L. F. Avila, E. F. Chillcce, L C. Barbosa, Univ. Estadual de Campinas (Brazil)

8257 1C Development of nanostructure based antireflection coatings for EO/ IR sensors [8257-53] A. K. Sood, R. E. Welser, A. W. Sood, Y. R. Puni, Magnolia Optic al Technologies, Inc. (United States); D. Poxson, J. Cho, E. F. Sc hubert, Rensselaer Polytechnic Institute (United Sta tes); N. K. Dhar, Defense Advanced Research Projects Agency (United States); M. B. Soprano, U.S. Amy Research, Development and Engineering Command (United States);

R. S. Balcerak, Consultant (United States)

8257 IE Middle infrared LEDs: key element for new generation chemical sensors [8257-55]

N. D. Stoya nov, K. M. Salikhov, K. V. Kalinina, B. E. Zhurtanov, S. S. Kizha ev, LED Mic rosensor NT, ШC (Russian Federation)

Author Index 
Proc. of SPIE Vol. $8257825701-8$

Downloaded From: https://www.spiedigitallibrary.org/conference-proceedings-of-spie on 26 Apr 2023 Terms of Use: https://www.spiedigitallibrary.org/terms-of-use 


\section{Conference Committee}

Symposium Chair

Klaus P. Streubel, OSRAM GmbH (Germany)

Symposium Cochairs

David L. Andrews, University of East Anglia Norwich (United Kingdom)

Liang-Chy Chien, Kent State University (United States)

Program Track Chair

James G. Grote, Air Force Research Laboratory (United States)

Conference Chairs

Shibin Jiang, AdValue Photonics, Inc. (United States)

Michel J. F. Digonnet, Stanford University (United States)

J. Christopher Dries, United Silicon Carbide, Inc. (United States)

Program Committee

Jean-Luc Adam, Université de Rennes 1 (France)

Norman C. Anheier, Jr., Pacific Northwest National Laboratory (United States)

Rolindes Balda, Universidad del País Vasco (Spain)

Robert P. Dahlgren, SETI Institute (United States)

Leonid B. Glebov, CREOL, The College of Optics and Photonics,

University of Central Florida (United States)

Min Gu, Swinburne University of Technology (Australia)

Seppo K. Honkanen, Aalto University School of Science and Technology (Finland)

Lili Hu, Shanghai Institute of Optics and Fine Mechanics (China)

Jacques Lucas, Université de Rennes 1 (France)

Yasutake Ohishi, Toyota Technological Institute (Japan)

Aydogan Ozcan, University of California, Los Angeles (United States)

Giancarlo C. Righini, Istituto di Fisica Applicata Nello Carrara (Italy)

Feng Song, Nankai University (China)

Setsuhisa Tanabe, Kyoto University (Japan)

John M. Zavada, National Science Foundation (United States) 
Session Chairs

1 Material Spectroscopy 1

Shibin Jiang, AdValue Photonics, Inc. (United States)

2 Material Spectroscopy II

Michel J. F. Digonnet, Stanford University (United States)

3 Laser Materials

Robert P. Dahlgren, SETI Institute (United States)

4 Microstructured Fibers

Robert P. Dahlgren, SETI Institute (United States)

$5 \quad$ Fiber Lasers

Seppo K. Honkanen, Aalto University School of Electrical Engineering (Finland)

6 Bragg Gratings

Shibin Jiang, AdValue Photonics, Inc. (United States)

7 Optical Components I

Aydogan Ozcan, University of California, Los Angeles (United States)

8 Optical Components II

Michel J. F. Digonnet, Stanford University (United States) 